\title{
Garlic Clove Catalyzed Biginelli Reaction in Water at Ambient Temperature
}

\author{
Hishame Abd-Elnabi ${ }^{1}$, Afaf Mohamed Abdel Hameed ${ }^{1}$, Ramadan Ahmed Mekheimer ${ }^{1,2}$, \\ Reham R. Awed ${ }^{1}$, Kamal Usef Sadek ${ }^{{ }^{*}}$ \\ ${ }^{1}$ Department of Chemistry, Faculty of Science, El-Minia University, El-Minia, Egypt \\ ${ }^{2}$ Department of Chemistry, Faculty of Science for Girls, King Abdulaziz University, Jeddah, KSA \\ Email: *kusadek@yahoo.com
}

Received September 2, 2013; revised October 2, 2013; accepted October 9, 2013

Copyright (C) 2013 Hishame Abd-Elnabi et al. This is an open access article distributed under the Creative Commons Attribution License, which permits unrestricted use, distribution, and reproduction in any medium, provided the original work is properly cited.

\begin{abstract}
A simple, green and highly efficient procedure for the Biginelli condensation reaction of aldehydes, $\beta$-ketoesters, urea or thiourea catalyzed by crushed garlic clove at ambient temperature is reported.
\end{abstract}

Keywords: Garlic Glove; Biocatalysis; Water; Biginelli Reaction

\section{Introduction}

Pyrimidines and their derivatives play an important role in human vital functions. The pyrimidine skeleton is the component of a series of natural compounds (vitamin $\mathrm{B}_{1}$, nucleic acids), chemotherapeutic drugs (Flurouracil) and synthetic medicines (Barbiturates). The biological importance of pyrimidine derivatives caused a significant interest in their synthesis [1-3]. Dihydropyrimidines (DHMPs) and their derivatives are pharmaceutically important as Calcium channel blockers, $\alpha_{1}-1-\mathrm{a}$-antagonists, antihypertensive agents, inhibitors of the fatty acid transporter, and mitotic kinesin inhibition [4,5]. These compounds have also been found to posses antiviral, antitumor and antibacterial properties [6]. Moreover, the biological activity of some isolated marine natural products and alkaloids have been attributed to the dihydropyrimidine moiety [7]. For example, the anti-cancer agent monostrol (Figure 1) has been shown to specifically effect mitosis via a new mechanism consisting of the specific and reversible inhibition of the motiliry of the motor protein mitotic kinesin [8-10].

Since more than one hundred year, Biginelli suggested a dihydropyrimidine ring construction based on the use of $\beta$-dicabonyl compounds as a source of two carbon fragment with aldehydes and urea or thiourea as a N-C-N fragment [11]. It is worth mentioning that Biginelli reac-

${ }^{*}$ Corresponding author. tion is one of the most named reactions and his collaboration is still considered one of the important pyrimidine synthesis. In the past 10 years, several one-pot methodologies for the synthesis of DHPM derivatives were developed and several modifications have been introduced. Most of them are based on Lewis acid-catalyzed reactions [12-19] which permits the reaction to proceed under milder conditions and with higher yields, than those outlined by Biginelli in the original procedure. Microwave irradiation has also proved beneficial [20]. Natural acidic catalysts have been also utilized [21]. Very recently, Biginelli reaction has been conducted under basic conditions. This involves the use of $\mathrm{PPh}_{3}$, under solvent free conditions [22], $t$ - $\mathrm{BuOK}$ at $70^{\circ} \mathrm{C}$ [23], chiral primary amines [24] and ammonium carbonate in water [25].

It is worth mentioning that many of these existing methods displayed drawbacks, such as environmental pollution caused by utilizing catalysts in stoichiometric<smiles>CCOC(=O)C1=C(C)NC(=S)NC1c1cccc(O)c1</smiles>

Figure 1. Monostrol. 
quantities, exotic reaction conditions, unsatisfactory yields and complicated operations while others posses some advantages overcoming these drawbacks. With the aim for the development of environmentally friendly technique, we investigated herein the utility of bio-catalysis in Biginelli reaction at ambient temperature. To the best of our knowledge, such approach has not been reported in the literature. Employing bio-catalysis is advantageous since it is green, proceeded at ambient temperature and is attractive from economical, environmental and handling point of view. In continuation of our work on the synthesis of azines and fused azines [26-28], we report herein the first attempt to synthesis DHMPs 4 using aryl aldehydes $\mathbf{1}$, ethyl acetoacetate $\mathbf{2}$, urea or thiourea 3 catalyzed by crushed garlic clove at ambient temperature (Scheme 1).

\section{Results and Discussion}

The structure of the products 4 were confirmed by comparison (TLC) with authentic samples [29-33], prepared by refluxing the reaction mixture in ethanol containing 1 $\mathrm{mL}$ of hydrochloric acid. In order to examine the effect of substituted aryl aldehyde $\mathbf{1}$ on the reaction rate and overall yield, various functionalized aryl aldehydes were used under the above reaction conditions. It has been found that the reaction proceeds smoothly to give DHMPs 4 in high yields with a slight decrease in the yield when the aryl substituent involves a strong electron donating group (cf. Table 1).

A proposed mechanism to account for the formation of $\mathbf{4}$ is demonstrated in Scheme $\mathbf{2}$ which involves enzymatic transformation of allin[(+)-(S)-allyl-cysteine-sulfoxide] influenced by alliinase producing allyl sulfenic acid, pyruvic acid and ammonia. Both could catalyze Biginelli reaction in the well established mechanism either acidic or basic conditions. At room temperature, these enzymatic transformations occur in $10-15$ minutes (Scheme 2).

\section{Conclusion}

We have successfully developed an easy, high yielding and versatile protocol for the synthesis of DHMPs from the reaction of aryl aldehydes, ethyl acetoacetate, urea or thiourea catalyzed by crushed garlic clove at ambient temperature. The process does not require the use of any hazardous or harmful catalysts and thus it is a simple, environmentally friendly technique of high atom economy and yields.

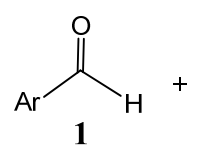<smiles>CCOC(=O)CC(C)=O</smiles><smiles>[X]C(N)=O</smiles><smiles>[X]C1NC(C)=C(C(=O)OCC)C([Y])N1</smiles>

\begin{tabular}{c|ll}
$\mathbf{1 , 4}$ & Ar & $\mathbf{X}$ \\
\cline { 2 - 3 } a & $\mathrm{C}_{6} \mathrm{H}_{5}$ & $\mathrm{O}$ \\
b & $4-\mathrm{MeO}-\mathrm{C}_{6} \mathrm{H}_{4}$ & $\mathrm{O}$ \\
c & $4-\mathrm{Me}-\mathrm{C}_{6} \mathrm{H}_{4}$ & $\mathrm{O}$ \\
d & $3-\mathrm{NO}_{2}-\mathrm{C}_{6} \mathrm{H}_{4}$ & $\mathrm{O}$ \\
e & $4-\mathrm{Cl}^{-} \mathrm{C}_{6} \mathrm{H}_{4}$ & $\mathrm{O}$ \\
$\mathrm{f}$ & $\mathrm{C}_{6} \mathrm{H}_{5}$ & $\mathrm{~S}$ \\
g & $2-\mathrm{MeO}_{2} \mathrm{C}_{6} \mathrm{H}_{4}$ & $\mathrm{~S}$ \\
h & $3-\mathrm{NO}_{2}-\mathrm{C}_{6} \mathrm{H}_{4}$ & $\mathrm{~S}$ \\
i & $4-\mathrm{Cl}-\mathrm{C}_{6} \mathrm{H}_{4}$ & $\mathrm{~S}$ \\
j & $4-\mathrm{MeO}-\mathrm{C}_{6} \mathrm{H}_{4}$ & $\mathrm{~S}$
\end{tabular}

Scheme 1. Synthesis of dihydropyrimidines.<smiles>C=CCC(=O)CC(N)C(=O)O</smiles>

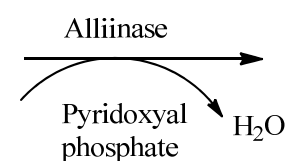<smiles>C=CCSO</smiles><smiles>CC(=O)C(=O)O</smiles>

Scheme 2. A proposed mechanism for the formation of dihydropyrimidines 4 . 
Table 1. Garlic clove synthesis of Biginelli DHMPs 4a-j.

\begin{tabular}{|c|c|c|c|c|c|c|}
\hline \multirow{2}{*}{ Product } & \multirow{2}{*}{$\mathrm{Ar}$} & \multirow{2}{*}{$\mathrm{X}$} & \multirow{2}{*}{ Time/h } & \multirow{2}{*}{ Yield } & \multicolumn{2}{|c|}{$\mathrm{Mp}\left({ }^{\circ} \mathrm{C}\right)$} \\
\hline & & & & & Found & Lit. \\
\hline $4 a$ & $\mathrm{C}_{6} \mathrm{H}_{5}$ & $\mathrm{O}$ & 3 & 92 & $202-203$ & $201-204$ [29] \\
\hline $4 b$ & 4-MeO- $\mathrm{C}_{6} \mathrm{H}_{4}$ & $\mathrm{O}$ & 2.5 & 85 & $203-205$ & $204-206$ [29] \\
\hline $4 c$ & 4-Me- $\mathrm{C}_{6} \mathrm{H}_{4}$ & $\mathrm{O}$ & 2.5 & 80 & $213-215$ & $212-213$ [29] \\
\hline $4 d$ & $3-\mathrm{NO}_{2}-\mathrm{C}_{6} \mathrm{H}_{4}$ & $\mathrm{O}$ & 3 & 75 & $225-227$ & $226-228$ [29] \\
\hline $4 e$ & 4-Cl- $\mathrm{C}_{6} \mathrm{H}_{4}$ & $\mathrm{O}$ & 3 & 83 & $212-214$ & $212-214[30]$ \\
\hline 4f & $\mathrm{C}_{6} \mathrm{H}_{5}$ & S & 2.5 & 90 & $207-209$ & $207-209$ [30] \\
\hline $4 g$ & $2-\mathrm{MeO}-\mathrm{C}_{6} \mathrm{H}_{4}$ & S & 1.5 & 83 & $190-191$ & $188-189$ [29] \\
\hline $4 h$ & $3-\mathrm{NO}_{2}-\mathrm{C}_{6} \mathrm{H}_{4}$ & $\mathrm{~S}$ & 2.5 & 74 & $210-212$ & $210-212[33]$ \\
\hline $4 i$ & 4-Cl- $\mathrm{C}_{6} \mathrm{H}_{4}$ & $\mathrm{~S}$ & 2 & 75 & $182-183$ & $180-182[33]$ \\
\hline $4 j$ & $4-\mathrm{MeO}-\mathrm{C}_{6} \mathrm{H}_{4}$ & S & 1.5 & 82 & $150-152$ & $148-150[31]$ \\
\hline
\end{tabular}

\section{Experimental}

\subsection{General}

Melting points were measured on a Gallenkamp apparatus and were not corrected. IR spectra were recorded with a Schimadzu 470 spectrophotometer in $\mathrm{KBr}$ disks. Peaks are reported in $\mathrm{cm}^{-1}$. ${ }^{1} \mathrm{H}$ and ${ }^{13} \mathrm{C}$ NMR spectra were recorded on a Bruker AM400 (400 MHz for ${ }^{1} \mathrm{H}$ NMR and $100 \mathrm{MHz}$ for ${ }^{13} \mathrm{C}$ NMR) spectrometer in DMSO$\mathrm{d}_{6}$ using TMS as an internal standard; the chemical shifts are given in $\delta$ units (ppm). Mass spectra were measured on a GCMS-QP1000EX (EI, $70 \mathrm{eV}$ ) mass spectrometer. Analytical thin-layer chromatography (TLC) was performed on aluminum sheets precoated with silica gel (Merck, Kieselgel 60F-254). Visualization was accomplished by UV light. Micro-analyses were performed at the Microanalytical Data Unit at Cairo University and analytical values obtained were within $\pm 0.4 \%$ of the calculated values.

\subsection{Synthesis of Dihydropyrimidines 4a-j; General Procedure}

To a mixture of each of aldehydes $\mathbf{1 a}-\mathbf{j}$ (10 $\mathrm{mmol})$, ethyl acetoacetate 2 (10 mmol) was added crushed garlic glove (100 mg every $15 \mathrm{~min}$.). Then, the reaction mixture was stirred at room temperature $\left(25^{\circ} \mathrm{C}\right)$ for the cited time (TLC) control. Then, the reaction mixture was dissolved in $\mathrm{EtOH}$, filtered and the filtrate was concentrated under reduced pressure. The resulting solid product was collected by filtration, dried and recrystallized from $\mathrm{EtOH}$.

\subsection{Ethyl 6-methyl-2-oxo-4-phenyl-1,2,3,4- tetrahydro-5-pyrimidine carboxylate (4a)}

${ }^{1} \mathrm{H}$ NMR (400 MHz, DMSO-d 6 ): $\delta=1.12(\mathrm{t}, J=7.2 \mathrm{~Hz}$, $3 \mathrm{H}), 2.33(\mathrm{~s}, 3 \mathrm{H}), 4.02(\mathrm{q}, J=7.2 \mathrm{~Hz}, 2 \mathrm{H}), 5.08(\mathrm{~d}, J=4$ $\mathrm{Hz}, 1 \mathrm{H}), 6.35$ (t, $J=7.5 \mathrm{~Hz}, 2 \mathrm{H}), 6.87$ (t, $J=7.3 \mathrm{~Hz}, 1 \mathrm{H})$, $7.33(\mathrm{~d}, J=7.5 \mathrm{~Hz}, 2 \mathrm{H}), 9.66(\mathrm{~s}, 1 \mathrm{H}), 10.44(\mathrm{~s}, 1 \mathrm{H}) .{ }^{13} \mathrm{C}$ NMR (100 MHz, DMSO-d $\left.\mathrm{d}_{6}\right): \delta=13.2,61.5,111.2$, $127.8,128.2$, 130.7, 158.5, 166.0. IR $(\mathrm{KBr}): v=3231$, 3207, 1689, $1664 \mathrm{~cm}^{-1}$. MS: $\mathrm{m} / \mathrm{z}=278\left(\mathrm{M}^{+}, 100\right)$. Found: C, 64.63; H, 6.22; N, 10.87; anal. calcd for $\mathrm{C}_{14} \mathrm{H}_{16} \mathrm{~N}_{2} \mathrm{O}_{3}$ : C, 64.60; H, 6.20; N, 10.76.

\subsection{Ethyl 4-(4-methoxyphenyl)-6-methyl-2-oxo- 1,2,3,4-tetrahydro-5-pyrimidine carboxylate (4b)}

${ }^{1} \mathrm{H}$ NMR (400 MHz, DMSO-d $\left.{ }_{6}\right): \delta=1.12(\mathrm{t}, J=7.2 \mathrm{~Hz}$, $3 \mathrm{H}), 2.22(\mathrm{~s}, 3 \mathrm{H}), 3.73(\mathrm{~s}, 3 \mathrm{H}), 4.20(\mathrm{q}, J=7.2 \mathrm{~Hz}, 2 \mathrm{H})$, $5.09(\mathrm{~d}, J=3.2 \mathrm{~Hz}, 1 \mathrm{H}), 6.88(\mathrm{~d}, J=8.4 \mathrm{~Hz}, 2 \mathrm{H}), 7.14$ (d, $J=8.4 \mathrm{~Hz}, 2 \mathrm{H}), 9.60$ (s, 1H), 10.15 (s, 1H). IR (KBr): v $=3230,3204,1688,1665 \mathrm{~cm}^{-1}$. MS: $\mathrm{m} / \mathrm{z}=290\left(\mathrm{M}^{+}\right.$, 100). Found: C, 61.96; H, 6.27; N, 9.59; anal. calcd for $\mathrm{C}_{15} \mathrm{H}_{18} \mathrm{~N}_{2} \mathrm{O}_{4}$ : C, 62.05; H, 6.24; N, 9.64.

\subsection{Ethyl 4-(4-methoxyphenyl)-6-methyl-2- thioxo-1,2,3,4-tetrahydro-5-pyrimidine carboxylate $(4 j)$}

${ }^{1} \mathrm{H}$ NMR (400 MHz, DMSO-d $\left.)_{6}\right): \delta=1.12(\mathrm{t}, J=7.1 \mathrm{~Hz}$, $3 \mathrm{H}), 2.29(\mathrm{~s}, 3 \mathrm{H}), 3.72(\mathrm{~s}, 3 \mathrm{H}), 4.00(\mathrm{q}, J=7.1 \mathrm{~Hz}, 2 \mathrm{H})$, $5.06(\mathrm{~d}, J=3.5 \mathrm{~Hz}, 1 \mathrm{H}), 6.92(\mathrm{~d}, J=8.5 \mathrm{~Hz}, 2 \mathrm{H}), 7.13(\mathrm{~d}$, $J=8.5 \mathrm{~Hz}, 2 \mathrm{H}), 9.22$ (s, 1H), 10.32 (s, 1H). IR (KBr): $v$ $=3230,3205,1688,1664 \mathrm{~cm}^{-1}$. MS: $\mathrm{m} / \mathrm{z}=306\left(\mathrm{M}^{+}\right.$, 100). Found: C, 58.76; H, 5.82; N, 9.23, S, 10.52; anal. calcd for $\mathrm{C}_{15} \mathrm{H}_{18} \mathrm{~N}_{2} \mathrm{O}_{3} \mathrm{~S}: \mathrm{C}, 58.80 ; \mathrm{H}, 5.92 ; \mathrm{N}, 9.14, \mathrm{~S}$, 10.47 .

\section{REFERENCES}

[1] Y. R. Mirzaei, H. Azamat and H. Namazi, "Investigation the Reactivity of Positions N-3, C-5 and $\mathrm{C}_{6}$-Methyl Group in Biginelli Type Compounds and Synthesis of New Dihydropyrimidine Derivatives," Journal of Heterocyclic Chemistry, Vol. 38, No. 5, 2001, pp. 1051- 
1054.

[2] M. R. Shaaban, T. S. Saleh, A. S. Mayhoub, A. Mansour and A. M. Farag, "Synthesis and Analgesic/Anti-Inflammatory Evaluation of Fused Heterocyclic Ring Systems Incorporating Phenylsulfonyl Moiety," Bioorganic \& Medicinal Chemistry, Vol. 16, No. 12, 2008, pp. 63446352.

[3] H. Cho, M. Ueda, K. Shima, A. Mizuno, M. Hayashimatsu, Y. Ohnaka, Y. Takeuchi, M. Hamaguchi, K. Aisaka and T. Hidaka, "Dihydropyrimidines: Novel Calcium Antagonists with Potent and Long-Lasting Vasodilative and Anti-Hypertensive Activity," Journal of Medicinal Chemistry, Vol. 32, No. 10, 1989, pp. 2399-2406. http://dx.doi.org/10.1021/jm00130a029

[4] G. C. Rovnyak, S. D. Kimball, B. Beyer, G.Cucinotta, J. D. DiMarco, J. Gougoutas, A. Hedberg, M. Malley, J. P. McCarthy, R. Zhang and S. J. Moreland, "Calcium Entry Blockers and Activators: Conformational and Structural Determinants of Dihydro-Pyrimidine Calcium Channel Modulators," Journal of Medicinal Chemistry, Vol. 38, No. 1, 1995, pp. 119-129.

http://dx.doi.org/10.1021/jm00001a017

[5] M. C. Van Zandt, M. L. Jones, D. E. Gunn, L. S. Geraci, J. H. Jones, D. R. Sawicki, J. Sredy, J. L. Jacot, A. Th. DiCioccio, T. Petrova, A. Mitschler and A. D. Podjarny, "Discovery of 3-[(4,5,7-Triflurobenzothiazol-2-yl)Methyl] Indole- $N$-Acetic Acid (Lidorestat) and Congeners as Highly Potent and Selective Inhibitors of Aldose Reeducates for Treatment of Chronic Diabetic Complications," Journal of Medicinal Chemistry, Vol. 48, No. 9, 2005, pp. 31413152 .

[6] T. Tsuruo, H. Iida, M. Nojiri, S. Tsukagoshi and Y. Sakurai, "Circumvention of Vincristine and Adriamycin Resistance in Vitro and in Vivo by Calcium Blockers," Cancer Research, Vol. 43, No. 6, 1983, pp. 2905-2910.

[7] B. B. Snider and Z. Shi, "Biometric Synthesis of (.+-.) Crambines A, B, C1 and C2. Revision of the Structure of Carbmines B and C1)," The Journal of Organic Chemistry, Vol. 58, No. 15, 1993, pp. 3828-3839.

[8] G. C. Rovnyak, S. D. Kimball, B. Beyer, G. Cucinotta, J. D. DiMarco, J. Gougoutas, A. Hedberg, M. Malley, J. P. McCarthy, R. Zhang and S. Moreland, "Calcium Entry Blockers and Activators: Conformational and Structural Determinants of Dihydro-Pyrimidine Calcium Channel Modulators," Journal of Medicinal Chemistry, Vol. 38, No. 1, 1995, pp. 119-129. http://dx.doi.org/10.1021/jm00001a017

[9] A. Dondoni, A. Massi and S. Sabbatini, "Improved Synthesis and Preparative Scale Resolution for Racemic Monastrol," Tetrahedron Letters, Vol. 43, No. 34, 2002, pp. 5913-5916.

[10] R. Laville, O. P. Thomas, F. Berrué, D. Marquez, J. Vacelet and $\mathrm{Ph}$. Amade, "Bioactive Guanidine Alkaloids from Two Caribbean Marine Sponges," Journal of Natural Products, Vol. 72, No. 9, 2009, pp.1589-1594. http://dx.doi.org/10.1021/np900244g

[11] P. Biginelli, "Derivati Aldeiduredici Degli Eteri Acetile Dossal-Acetico," Gazzetta Chimica Italiana, Vol. 23, 1893, pp. 360-416.
[12] A. S. Paraskar, G. K. Dewkar and A. Sudalai, "Cu(OTf $)_{2}$ a Reusable Catalyst for High-Yield Synthesis of 3,4-Dihydropyrimidin-2(1H)-Ones," Tetrahedron Letters, Vol. 44, No. 16, 2003, pp. 3305-3308. http://dx.doi.org/10.1016/S0040-4039(03)00619-1

[13] G. Sabitha, K. B. Reddy, J. S. Yadav, D. Shailaja and K. S. Sivudu, "Ceria/Vinyl pyridine Polymer Nanocomposite: An Ecofriendly Catalyst for the Synthesis of 3,4-dihydropyrimidin-2(1H)-ones," Tetrahedron Letters, Vol. 46, No. 47, 2005, pp. 8221-8222. http://dx.doi.org/10.1016/j.tetlet.2005.09.100

[14] J. Azizian, A. A. Mohammadi, A. R. Karimi and M. R. Mohammadizadeh, "KAl $\left(\mathrm{SO}_{4}\right) \cdot 12 \mathrm{H}_{2} \mathrm{O}$ Supported on Silica Gel as a Novel Heterogeneous System Catalyzed Biginelli Reaction: One-Pot Synthesis of Dihydropyrimidinones Under Solvent-Free Conditions," Applied Catalysis A: General, Vol. 300, No. 1, 2006, pp. 85-88.

[15] K. U. Sadek, F. Al-Qalaf, M. M. Abdelkhalik and M. H. Elnagdi, "Cerium (IV) Ammonium Nitrate as an Efficient Lewis Acid for One-Pot Synthesis of 3,4-Dihydropyrimidin-2(1H)-ones and their Corresponding 2(1H)-thiones," Journal of Heterocyclic Chemistry, Vol. 47, No. 2, 2010, pp. 284-286. http://dx.doi.org/10.1002/jhet.259

[16] N. Shapiro and A. Vigalok, "Highly Efficient Organic Reactions 'on Water' and 'in Water' and Both," Angewandte Chemie, Vol. 120, No. 15, 2008, pp. 2891-2894. doi: 10.1002/ange. 200705347.

[17] S. D. Salim and K. G. Akamanchi, "Sulfated Tungstate: An Alternative, Eco-Friendly Catalyst for Biginelli Reaction," Catalysis Communications, Vol. 12, No. 12, 2011, pp. 1153-1156. doi: org/10.1016/j.catcom.2011.02.018.

[18] S. Chitra and K. Pandiarajan, "Calcium Fluoride: An Efficient and Reusable Catalyst for the Synthesis of 3,4Dihydropyrimidin-2(1H)-ones and Their Corresponding 2-(1H)-thiones: An Improved High Yielding Protocol for Biginelli Reaction," Tetrahedron Letters, Vol. 50, No. 19, 2009, pp. 2222-2224.

[19] P. G. Mandhane, R. S. Joshi, D. R. Nagargoje and C. H. Gill, "An Efficient Synthesis of 3,4-Dihydropyrimidin2(1H)-ones Catalyzed by Thiamine Hydrochloride in Water Under Ultrasound Irradiation," Tetrahedron Letters, Vol. 51, No. 23, 2010, pp. 3138-3140.

[20] K. K. Pasunooti, H. Chai, C. N. Jensen, B. K. Gorityala, S. Wang and X.-W. Liu, "A Microwave-Assisted, Copper Catalyzed three-component Synthesis of Dihydro-Pyrimidinones under Mild Conditions," Tetrahedron Letters, Vol. 52, No. 1, 2011, pp. 80-84.

[21] S. Patil, S. D. Jodhav and M. B. Deshmukh, "Natural Acid Catalyzed Multi-Component Reactions as a Green Approach," Archives of Applied Science Research, Vol. 3, No. 1, 2011, p. 203. www.scholarresearchlibirary.com

[22] A. Debache, M. Amimour, A. Belfaitah, S. Rhouati and B. Carboni, "A One-Pot Biginelli Synthesis of 3,4-Dihydropyrimidin-2(1H)-Ones/Thiones Catalyzed by Triphenylphosphine as Lewis Base," Tetrahedron Letters, Vol. 49, No. 42, 2008, pp. 6119-6121.

[23] Z.-L. Shen, X.-P. Xu and S.-J. Ji, "Bronsted Base-Catalyzed One-Pot Three Component Biginelli Type Reaction: An Efficient Synthesis of 4,5,6-Triaryl-3,4-Dihydropyri- 
midin-2(1H)-one and Mechanistic Study," The Journal of Organic Chemistry, Vol. 75, No. 4, 2010, pp. 1162-1167. http://dx.doi.org/10.1021/jo902394y

[24] D. Ding and C.-G. Zhao, "Primary Amine Catalyzed Biginelli Reaction for the Enantioselective Synthesis of 3,4-Dihydropyrimidin-2(1H)-ones," European Journal of Organic Chemistry, Vol. 2010, No. 20, 2010, pp. 38023805. http://dx.doi.org/10.1002/ejoc.201000448

[25] F. Tamaddon, Z. Razmi and A. A. Jafari, "Synthesis of 3,4-Dihydropyrimidin-2(1H)-ones and 1,4-Dihydropyridines Using Ammonium Carbonate in Water," Tetrahedron Letters, Vol. 51, No. 8, 2010, pp. 1187-1189.

[26] R. A. Mekheimer, A. M. Abdel Hameed and K. U. Sadek, "Solar Thermochemical Reactions: Four-Component Synthesis of Polyhydroquinoline Derivatives Induced by Solar Thermal Energy," Green Chemistry, Vol. 10, No. 5, 2008, pp. 592-593.

[27] K. U. Sadek, R. A. Mekheimer, A. M. Abdel Hameed, F. Elnahas and M. H. Elnagdi, "Green and Highly Efficient Synthesis of 2-Arylbenzothiazoles Using Glycerol without Catalyst at Ambient Temperature," Molecules, Vol. 17, No. 5, 2012, pp. 6011-6019. http://dx.doi.org/10.3390/molecules17056011

[28] K. U. Sadek, R. M. Shaker, M. AbdElrady and M. H. Elnagdi, " A Novel Method for the Synthesis of Polysubstituted Diaminobenzonitrile Derivatives Using Controlled Microwave Heating," Tetrahedron Letters, Vol. 51,
No. 48, 2010, pp. 6319-6321.

[29] C.-J. Liu and J.-D. Wang, “Copper (II) Sulfamate: An Efficient Catalyst for One-Pot Synthesis of 3,4-Dihydropyrimidin-2(1H)-ones and Thiones," Molecules, Vol. 14, No. 2, 2009, pp. 763-770. http://dx.doi.org/10.3390/molecules14020763

[30] J. S. Yadav, B. V. S. Reddy, K. B. Reddy, K. S. Raj and A. R. Prasad, "Ultrasound-Accelerated Synthesis of 3,4Dihydropyrimidin-2(1H)-ones with Ceric Ammonium Nitrate," Journal of the Chemical Society, Perkin Transactions, Vol. 1, No. 16, 2001, pp. 1939-1941. http://dx.doi.org/10.1039/b102565c

[31] H. W. Zhan, J. X. Wang and X. T. Wang, "Solvent- and Catalyst-Free Synthesis of Dihydropyrimidinthiones in One-Pot under Focused Microwave Irradiation," Chinese Chemical Letters, Vol. 19, No. 10, 2008, pp. 1183-1185.

[32] A. D. Patil, N. V. Kumar, W. C. Kokke, M. F. Bean, A. J. Freyer, C. D. Brosse, S. Mai, A. Truneh, D. J. Faulkner, B. Carte, A. L. Breen, R. P. Hertzberg, R. K. Johnson, J. W. Westly and B. C. M. Potts, "Novel Alkaloids from the Sponge Batzellasp: Inhibitors of HIV gp120-Human CD4 Binding," European Journal of Organic Chemistry, Vol. 60, No. 7, 1995, pp.1182-1188.

[33] R. Ghosh, S. Maiti and A. Chakraborty, "In(OTf) $)_{3}$-Catalyzed One-Pot Synthesis of 3,4-Dihydropyrimidin-2(1H)ones," Journal of Molecular Catalysis A: Chemical, Vol. 217, No. 1, 2004, pp. 47-50. 